\title{
Attitudes of graduating Canadian urology residents on the job market: Is it getting better or are we just spinning our wheels?
}

Gregory William Hosier; Naji Touma

Queen's University, Kingston, ON, Canada

Cite as: Can Urol Assoc J 2017 Dec. 22; Epub ahead of print. http://dx.doi.org/10.5489/cuaj.4765

Published online December 22, 2017

$* * *$

\section{Abstract}

Introduction: There has been increasing awareness of employment difficulties for physicians, especially surgeons, in Canada over the past few years. Our objective was to elucidate the attitudes and experiences of graduating Canadian urology residents in obtaining employment. Methods: We surveyed four separate cohorts of graduating urology residents in 2010, 2011, 2016, and 2017. Responses from the 2010 and 2011 cohorts were combined and compared to the combined results of the 2016 and 2017 cohorts. Mean Likert responses were compared using unpaired t-tests. An agreement score was created for those responding with "strongly agree" and "agree" on the Likert scale.

Results: A total of 126 surveys were administered with a $100 \%$ response rate. The job market was rated as poor or very poor by $64.9 \%$ and $58.4 \%$ of graduates in 2010/2011 and 2016/2017, respectively ( $\mathrm{p}=0.67$ ). Lack of resources was identified as the biggest barrier to improved employment in both cohorts. Networking at meetings and staff urologists at their institution were the most important factors aiding employment identified by both cohorts. The ideal practice was academic or academically associated community practices in a large urban area with 5-10 partners for both cohorts.

Conclusions: The majority of graduating urology residents viewed the job market as poor or very poor and this did not change over a six-year period. It is unclear how much personal preference for location and practice type drove the somewhat negative outlook of employment opportunities, as the majority of residents were seeking large urban, academic, or academically associated community practices in competitive locations. 


\section{Introduction}

There has been increasing awareness of employment difficulties for physicians in Canada over the past few years. This was highlighted in a 2013 report on physician employment by the Royal College of Physicians and Surgeons of Canada, which found that $16 \%$ of all new Royal College certificants were unable to find employment. ${ }^{1}$ It was hypothesized that a significant contributing factor to employment difficulties at the time was delayed retirement of specialists because of poor stock performance in wake of the 2008 recession. Other factors impacting the job market identified in this report included decreased hospital funding, increasing use of physicianassistants and nurse practioners, limited career counseling during residency, and personal factors (inability to relocate due to family, etc.).

In particular, employment opportunities seem to be limited in the surgical disciplines and other resource intensive specialties. According to a survey of Resident doctors of Canada in 2012, only $14 \%$ of surgical residents said they were confident in finding employment compared to $30 \%$ of residents in a medical specialty and $80 \%$ of residents in family medicine. ${ }^{2}$ According to the Royal College survey, 30\% of those in surgical disciplines pursued fellowship training because they were unable to find a job compared to $16 \%$ of all specialists. Together these data paint a disconcerting picture of the employment landscape for surgical disciplines in Canada.

To date, there has been only limited data on the job landscape within Canadian urology. Within the Royal College survey, $40 \%$ of Urology residents responded that they were unable to find employment. However, the response rate was only $26 \%$ and it is possible that a significant sample bias was present. A human resource assessment of academic urology positions revealed that there was an expected need of 68 academic positions over the period of 2012-2017. ${ }^{3}$ Using computer modeling, Pace et al. predicted that by 2010 there would be a shortage of 101 urologists in Ontario. ${ }^{4}$ Of graduating Canadian urology residents between 1998 and 2009 over $98 \%$ were employed with $16 \%$ practicing in the United States. ${ }^{5}$ It is unclear how the Royal College survey results fit with the findings of the above studies. Given that a significant contributing factor to poor employment rates was believed to be the 2008 recession, it is also unclear whether employment statistics have improved more recently.

Our objective was to elucidate the attitudes and experiences informing the pursuit of employment of Canadian urology residents. By comparing cross-sections 6 years apart, we aimed to identify important trends to inform career planning for post-graduate urology programs in Canada. We hypothesized that attitudes regarding the job market among graduates in the 2016/2017 cohort may differ from graduates in 2010/2011 as fewer graduates in 2016/2017 would be expected to feel the impact of delayed retirement.

\section{Methods}

We surveyed graduating Urology residents at the time of the annual Queen's Urology Examination Skills Training Program (QUEST) in Kingston, Ontario. To evaluate job trends over time, we compared results of a combined cohort consisting of graduates in 2010 and 2011 to a combined cohort of graduates in 2016 and 2017. The questionnaire was administered to 
willing participants in an auditorium at the beginning of the program. All survey responses were anonymous. We chose these participants as a convenience sample and because they would most likely be entering the urology job market in the next few years. Approval was obtained from the Queen's University institutional review board with assurance of confidentiality provided to all participants.

The questionnaire consisted of 20 open- and close-ended questions exploring residents' outlook on the urology job market and plans after completion of residency using: 1) 5-point Likert scale, 2) "yes" or "no" answers, and 3) categorical answers. The anchoring score of 3 on the Likert scale was described as "neutral" on the survey, while " 1 " and "2" corresponded to "strongly disagree" and "disagree", and " 4 " and " 5 " corresponded to "agree" and strongly "agree", respectively. The questionnaire was developed specifically for this survey and resulted from previous experiences with survey construction..$^{6-8} \mathrm{~A}$ limited number of residents and educators involved in postgraduate programs were asked to assess and modify the survey for clarity.

Responses to the questions using the 5-point Likert scale are described as means \pm standard deviation (SD). For ease of reporting and to distinguish trends in the intensity of the survey responses, an agreement score combining the responses of 4 (agree) and 5 (strongly agree) was created and reported alongside the mean 5 -point Likert score. ${ }^{9}$ Likert scale responses between 2010/2011 and 2016/2017 graduates were compared using unpaired t-tests. Categorical data was compared using Chi-square tests. A two-sided $\mathrm{P}$ value of $<0.05$ was the threshold for statistical significance in all comparisons. Analyses were performed using GraphPad Prism 7.

\section{Results}

A total of 126 surveys (58 to 2010/2011 and 68 to 2016/2017 graduating residents) were administered with a 100\% response rate. Of those graduating in 2010/2011, 86\% were planning fellowship training, $12.3 \%$ had secured a staff position in Canada, and $1.7 \%$ a staff position in the USA. Of those graduating in 2016/2017, 79\% were planning fellowship training, $14.7 \%$ obtained a staff position in Canada, $2.9 \%$ a staff position in the USA, $1.5 \%$ an international staff position, and $1.5 \%$ a locum. Of those graduating in 2010/2011, $19.3 \%$ and $14 \%$ said they were offered an academic or community staff position contingent on them completing a fellowship, respectively. Of those graduating in 2016/2017, $11.8 \%$ and $1.5 \%$ said they were offered an academic or community staff position contingent on them completing a fellowship, respectively.

The job market was rated as poor or very poor by $64.9 \%$ and $58.4 \%$ of graduates in 2010/2011 and 2016/2017 respectively. Views on the state of the job market in Canada were probed with plausible, close-ended statements and candidates rated their answers with a 5-point Likert scale (Fig. 1). The most commonly cited statement regarding the job market in Canada was that there is a need in Canada for urologists but hospital resources are lacking. This was not different from 2010/2011 to 2016/2017 with 79.3\% and 89.7\% agreeing with this statement and a mean Likert score of 4.3 ( \pm 0.9 SD) and 4.5 ( \pm 0.7 ) respectively ( $\mathrm{P}=0.21$ ). In both the 2010/2011 and 2016/2017 cohorts, the highest agreement for ways to aid job employment was to increase 
hospital resources and provincial funding for urological services with $89.5 \%$ and $91 \%$ agreement, and mean Likert scores of $4.51 \pm 0.88$ and $4.66 \pm 0.64$, respectively ( $\mathrm{P}=0.31$; Fig. 2).

When asked to rate how helpful various sources were in finding a job in urology, graduates in 2010/2011 ranked networking at meetings as the most important source (75.9\% agreement, mean Likert score $4.1 \pm 1.03$ ), while graduates in 2016/2017 ranked staff urologists at their institution as the most important source (77.6\% agreement, mean Likert score of $4.0 \pm 1.14$; Fig. 3). When asked if they were aware of Canadian Urology Association (CUA) initiatives to identify urology jobs, $17.2 \%$ and $14.7 \%$ of graduates in 2010/2011 and 2016/2017 respectively responded that they were. Of those graduating in 2010/2011 and 2016/2017, 41.4\% and 20.6\% were aware of American Urology Association (AUA) initiatives to identify jobs respectively.

When asked to rate the importance of various factors in choosing a urology position, the most important factor for both graduates in 2010/2011 and 2016/2017 was comfort with colleagues (93.2\% agreement, mean Likert score of $4.5 \pm 0.79$; 91\% agreement, mean Likert score 4.4 \pm 0.74 respectively; $\mathrm{P}=0.33$; Fig. 4).

Among the 2010/2011 cohort, the most common response when asked about the ideal practice type, community size, and number of partners, was academic with teaching emphasis (39\%), large urban (51.7\%), and 5-10 partners (65\%) respectively. Among the 2016/2017 cohort, the most commonly reported ideal practice type, community size, and number of partners was community with academic affiliation (42.6\%), large urban (48.5\%), and 5-10 partners (47.8\%).

Figure 5 shows the home location and desired practice location for graduates in 2010/2011 and 2016/2017. Residents were asked to identify their home province without providing a specific definition but it was implied that it would be a location where they spent a significant portion of their youth. In both the 2010/2011 and 2016/2017 cohorts, provinces with net immigration included British Columbia, and Alberta. Provinces with net emigration were Saskatchewan/Manitoba, Quebec, and Newfoundland. Provinces with stability were Ontario, and Atlantic provinces.

Of the $2010 / 2011$ and $2016 / 2017$ graduates, $66.7 \%$ and $73.1 \%$ completed community elective rotations. In those who did community electives, $>50 \%$ agreed it was essential to improve surgical skills and $>50 \%$ felt they would have benefitted from additional community elective time. While $86.2 \%$ and $90.9 \%$ felt they had enough exposure to academic urology for career planning, only $56.9 \%$ and $60.6 \%$ felt they had adequate exposure to community urology for career planning in the 2010/2011 and 2016/2017 cohorts, respectively.

\section{Discussion}

The results of this study indicate that the majority of final year Urology residents viewed the job market as poor or very poor and this did not change over a 6-year period. Of those graduating in 2010/2011 and 2016/2017, 86\% and 79\% went on to pursue fellowship training, while $12.3 \%$ and $14.7 \%$ went directly into community practice, respectively. Rates of fellowships were similar to those previously reported for Canadian urology. ${ }^{5,8}$ The low rate of graduates entering 
the workforce upon completion of residency is likely multifactorial. It includes factors such as the increasing complexity of urological practice and the need for sub-specialization, the interests of graduates, described here, in pursuing large practices in urban centres. However, it likely does also include the negative perceptions - possibly rooted in experience - of graduates of the Canadian urology job market. It is important to point out that these rates of fellowship and direct entry into community practice were similar to otolaryngology. ${ }^{10}$

Lack of resources was identified as the biggest barrier to improved employment in both the 2010/2011 and 2016/2017 cohorts. In a 2012 survey of all residents across Canada, 74.5\% reported finding employment at the end of residency very or somewhat stressful. ${ }^{2}$ Respondents similarly identified lack of resources as the most important barrier to improved employment. Lack of hospital resources was a key driver of under- and un-employment in surgical and resource-intensive specialties identified in the 2013 Royal College report. ${ }^{1}$ According to the Canadian Institute of Health Information, health care spending between 2010 and 2016 decreased by $0.1 \%$ per year, despite an increasing and aging population. ${ }^{10}$ Hospital funding, which drives employment of resource-intensive, hospital based-physicians, like urologists, has seen the lowest rate of growth since the late 1990s, increasing by 3.4\%, 2.5\%, and $2 \%$ in 2012, 2013, and 2014 respectively. ${ }^{11}$ Consequently, there is some evidence to support the attitudes of graduating urology residents in the current study that lack of resources may be limiting employment opportunities for them in Canada.

Another key driver of under- and un-employment identified in the 2013 Royal College report was delayed retirement due to poor stock market performance following the 2008 recession. We hypothesized that attitudes regarding the job market among graduates in the 2016/2017 cohort may differ from graduates in 2010/2011 as fewer graduates in 2016/2017 would be expected to feel the impact of delayed retirement. Interestingly, there were no differences in attitudes regarding employment between the 2010/2011 and 2016/2017 graduates. When asked whether they felt a mandatory retirement age would improve employment, there was poor agreement among the 2010/2011 and 2016/2017 cohorts. However, there is some evidence to indicate that delayed retirement or semi-retirement may be limiting employment opportunities for new urology graduates in Canada. According to the Canadian Medical Association (CMA) specialty profile on urology, there were 37 retirees (defined as giving up license) between 2013 and 2015. ${ }^{12}$ This was much lower than the 93-urology residents who graduated during this timeframe. ${ }^{13}$ Throughout this time period, the overall number of practicing urologists in Canada remained largely stable at around 680-690. ${ }^{12}$ This steady-state level of urologists in Canada is maintained possibly by a proportion of newly graduated and fellowshiptrained urologists utilizing locums, morphed practices, or moving abroad.

A previous work force planning study for Ontario urologists published in 1999 by Pace and colleagues estimated that there would be a shortage of urologists in Ontario by 2010 unless training spots were increased. ${ }^{4}$ In response to this and increasing numbers of medical school trainees, there was an increase in urology residency spots from the historic number of 15 to the 
current number of 33 throughout the early 2000's. This increase was largely driven by the Ontario programs through expansion as well as the addition of one new training program. Currently, the number of practicing urologists in Ontario (255) is similar to the predicted number of required urologists reported by Pace et al. (259). ${ }^{4}$ Why then is there a perceived lack of jobs in the graduating cohorts? Whereas the mathematical model used by Pace et al. assumed that one fifteenth of the group of urologists aged 55 to 70 years would retire annually, and all urologists beyond age 70 would retire, the model did not account for semi-retirement or retirement beyond age 70 that is believed to be occurring now. Additionally, as discussed above, despite increased numbers of graduating residents, there has not been a concomitant increase in hospital resources. Together, these factors may explain some of the discrepancy between the previously anticipated shortage of urologists in Ontario and the poor employment outlook of graduating urology residents in the current study. A previous report of program directors indicated that all 258 urology graduates between 1998 and 2009 are employed with only a 16\% attrition rate to the United States. ${ }^{5}$ However, the increase in the available training positions did not really translate into graduates till the later part of the 2000s. It is therefore conceivable that the job prospects of current graduates are more precarious.

Given the poor job outlook reported here, one solution may be to reduce the number of urology training positions in Canada. However, it is unclear if the current population-to-urologist ratio is optimal given the long wait times for urological procedures and the fact that the population-to-urologist ratio has not changed over the past 20 years despite an aging population. Furthermore, if we contract training programs because of this perceived lack of employment opportunity for recently graduated urologists, current academic centres may not be able to keep up with the growing clinical service demands. This may be further exacerbated by the start of competency based medicine in which there is more emphasis placed on skill acquisition for residents as opposed to simply providing service. One solution to meeting the service needs may be to increase utilization of hospitalists or similar physician extenders to fill the service gap. Increased utilization of fellows may be another mechanism that is allowing increasing service needs to be met. Despite the negative outlook of the job market by the current graduating residents, more information is needed about the impact of decreasing the number of trainees in the context of current service demands and the anticipated population needs for urological care.

When seeking out employment, the two most helpful resources reported by graduates were networking at meetings and staff urologists at their institutions. In a small discipline like urology, it makes intuitive sense that face-to-face meetings would play an important role in facilitating employment by allowing discussion of potential unofficial job openings, facilitating arrangement of electives, and allowing for collegial relationships to develop. Indeed, we found that comfort with partners was the most important factor to graduating residents seeking employment and it is likely that this sentiment goes both ways. Very few respondents were aware of any CUA or AUA initiatives to identify jobs in Canada or the USA respectively. At the time of writing this manuscript, the authors were aware of classified postings in the CUAJ and 
on the CUAJ website as well as an AUA job finder online tool. It is possible that with direct probing a larger proportion of graduates would have reported being aware of these tools. Nevertheless, this finding indicates a possible unfulfilled need that could be addressed by the CUA and AUA.

The majority of graduates in the 2010/2011 and 2016/2017 cohorts wanted to practice in large cities (population $>500,000$ ), with 5-10 urology partners, at academic centres. According to the CMA, only $29 \%$ of urologists across Canada practice at academic hospitals compared to the $59.3 \%$ and $55.9 \%$ of 2010/2011 and 2016/2017 graduates who desired positions at academic centres. ${ }^{12}$ A detailed survey of chairmen of the 13 academic centres with residency programs in Canada identified a need for 68 urologists over a 5-year period from 2012 to 2017. ${ }^{3}$ This is compared to the total of 70 graduates in the combined 2010/2011 and 2016/2017 cohorts (4 year period only) who desired to practice at academic centres. These academic centres in large part also represent the urology groups in large urban centres with $>10$ or 5-10 partners. Consequently, there seems to be a discrepancy between the available and desired practice types, sizes and locations for graduating urology residents. It is natural that graduates would be interested in remaining in larger communities where they have conducted their residency and medical training. Along with urological training, trainees, often in their 20s and 30s, are laying down roots and developing relationships within these communities. This would seem to argue for improved integration of community practice exposure within residency.

Our study has several limitations. The results are derived from a self-report survey on the experiences and attitudes of graduating residents in urology and any independent verification of data was not possible. The survey was created specifically for this cohort of trainees in Canada and has not been independently validated. Finally, the survey relied mostly on close-ended, Likert score questions with inherent limitations in interpretation and analysis in describing attitudes. However, the high overall response rates and the consistency of the responses over the 4 years would suggest some degree of assurance of the validity of the observations from the survey.

\section{Conclusion}

This study indicates that the majority of graduating urology residents viewed the Canadian job market as poor or very poor and this did not change over a 6-year period. Lack of resources was identified as the biggest barrier to improved employment in both the 2010/2011 and 2016/2017 cohorts. Overall, it is unclear how much personal preference for location and practice type drove the somewhat negative outlook of employment opportunities, as the majority of residents were seeking large, urban, academic or academically associated community practices, in competitive locations. 


\section{References}

1. Frechette D, Hollenberg D, Schrichand A, et al. What's really behind Canada's unemployed specialists? Too many, too few few doctors? Findsings from the Royal College's employment study. Ottawa, Ontario; 2013.

http://www.royalcollege.ca/rcsite/documents/health-policy/employment-report-2013e.pdf

2. The Royal College of Physicians and Surgeons of Canada. National Physician Survey, Results for Medical Residents. 2012. http://nationalphysiciansurvey.ca/wpcontent/uploads/2013/02/NPS2012-Resident-Full-EN.pdf

3. Bachir BG, Aprikian AG, et al. Human resource assessment of academic urology across Canada: What are the future job prospects? Can Urol Assoc J 2013;7:162. Available from: http://www.ncbi.nlm.nih.gov/pubmed/23826042

4. Pace KT, Provan JL, Jewett MA. The urology work force in Ontario for the 21st century: feast or famine? Can J Surg 1999;42:181-9. http://www.ncbi.nlm.nih.gov/pubmed/10372014

5. Welk B, Kodama R, Macneily A. The newly graduated Canadian urologist: Overtrained and underemployed? Can Urol Assoc J 2013;7:E10-5. http://www.ncbi.nlm.nih.gov/pubmed/23401733

6. Roth K, Siemens DR. The status of evidence-based medicine education in urology residency. Can Urol Assoc J 2010;4:114-20. http://www.ncbi.nlm.nih.gov/pubmed/20368894

7. Roberts G, Beiko D, Touma N, et al. Are we getting through? A national survey on the CanMEDS communicator role in urology residency. Can Urol Assoc J 2013;7:437-41. http://www.ncbi.nlm.nih.gov/pubmed/24381664

8. Touma NJ, Siemens DR. Attitudes and experiences of residents in pursuit of postgraduate fellowships: A national survey of Canadian trainees. Can Urol Assoc J 2014;8:437. http://cuaj.ca/index.php/journal/article/view/2136

9. Sullivan GM, Artino AR, Jr. Analyzing and interpreting data from likert-type scales. J Grad Med Educ 2013;5:541-2. http://www.ncbi.nlm.nih.gov/pubmed/24454995

10. Brandt MG, Scott GM, Doyle PC, et al. Otolaryngology - Head and Neck Surgeon unemployment in Canada: a cross-sectional survey of graduating Otolaryngology Head and Neck Surgery residents. J Otolaryngol - Head Neck Surg 2014;43:37. http://www.ncbi.nlm.nih.gov/pubmed/25683630

11. Canadian Institute of Health Information. National Health Expenditure Trends 1975 to 2016. Ottawa, Ontario; 2016. https://secure.cihi.ca/free_products/NHEX-TrendsNarrative-Report_2016_EN.pdf

12. Canadian Medical Association. Specialty profile, Urology. 2016. https://www.cma.ca/Assets/assets-library/document/en/advocacy/profiles/urology-e.pdf

13. Canadian Residency Matching Service. Data and Reports, CaRMS. 2016. http://www.carms.ca/en/data-and-reports/r-1/ 


\section{Figures and Tables}

Fig. 1. Views on the urology job market in Canada were not different between 2010/2011 and 2016/2017 final year residents.

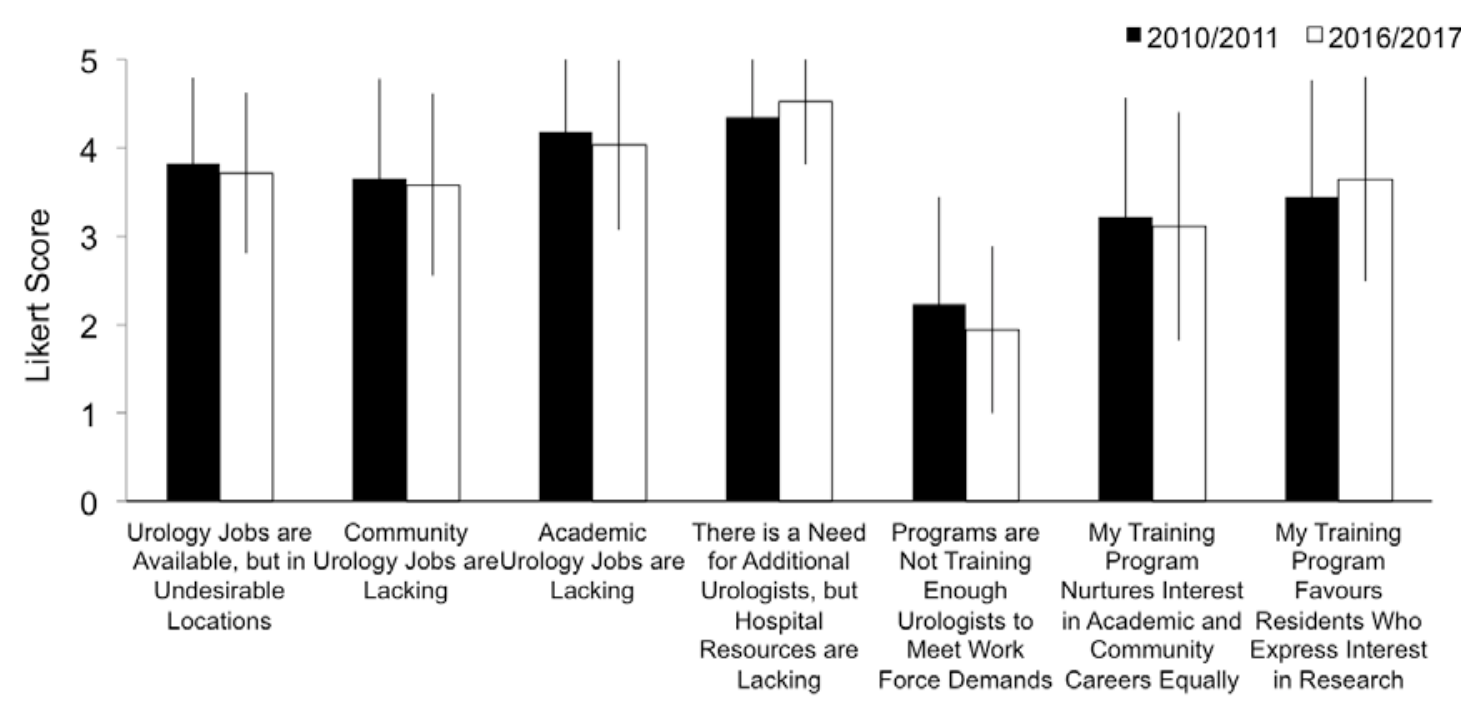

Fig. 2. Views on how to aid job employment were not different between 2010/2011 and 2016/2017 final year residents.

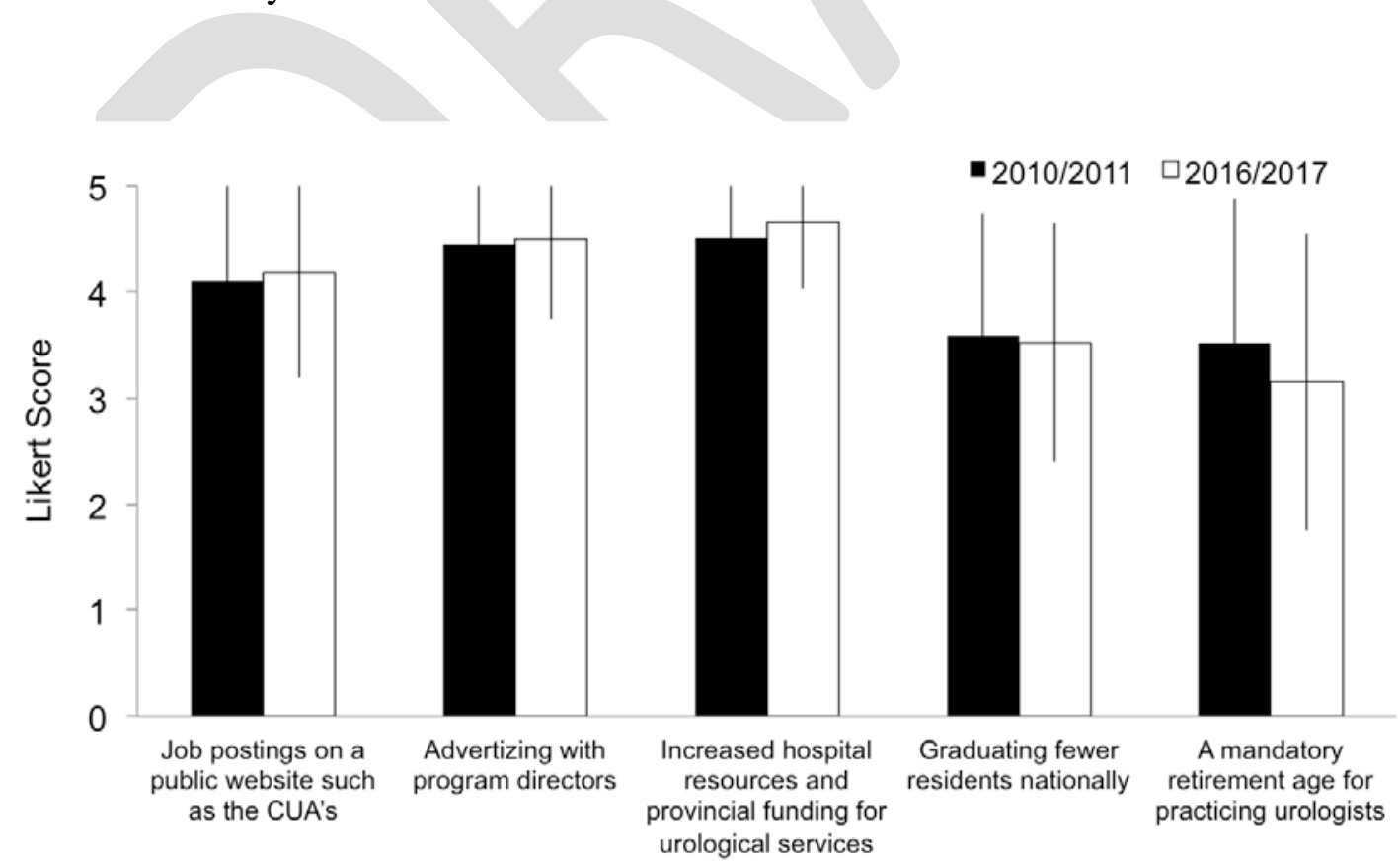


Fig. 3. Helpful resources to find employment were not different among 2010/2011 and 2016/2017 final year residents.

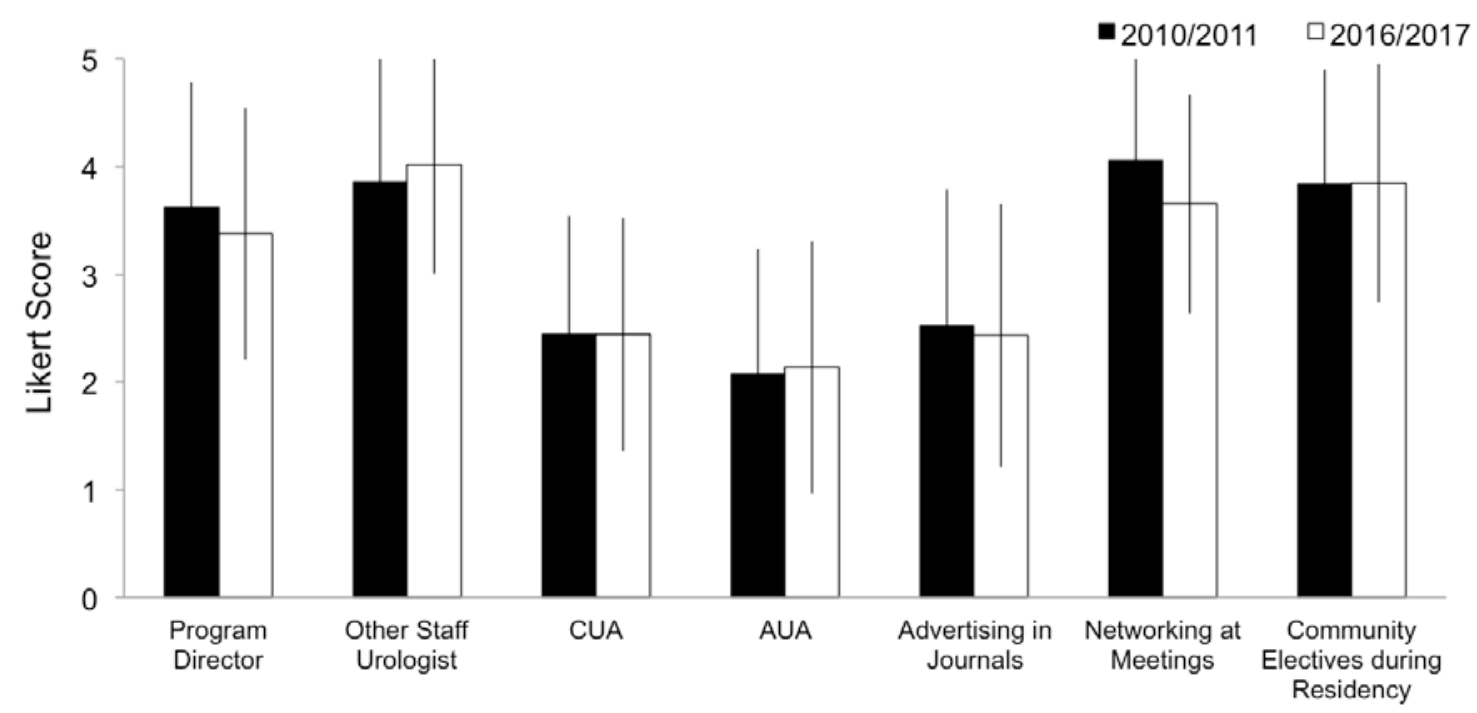

Fig.4. Factors considered when seeking a urology position were not different among 2010/2011 and 2016/2017 final year residents.

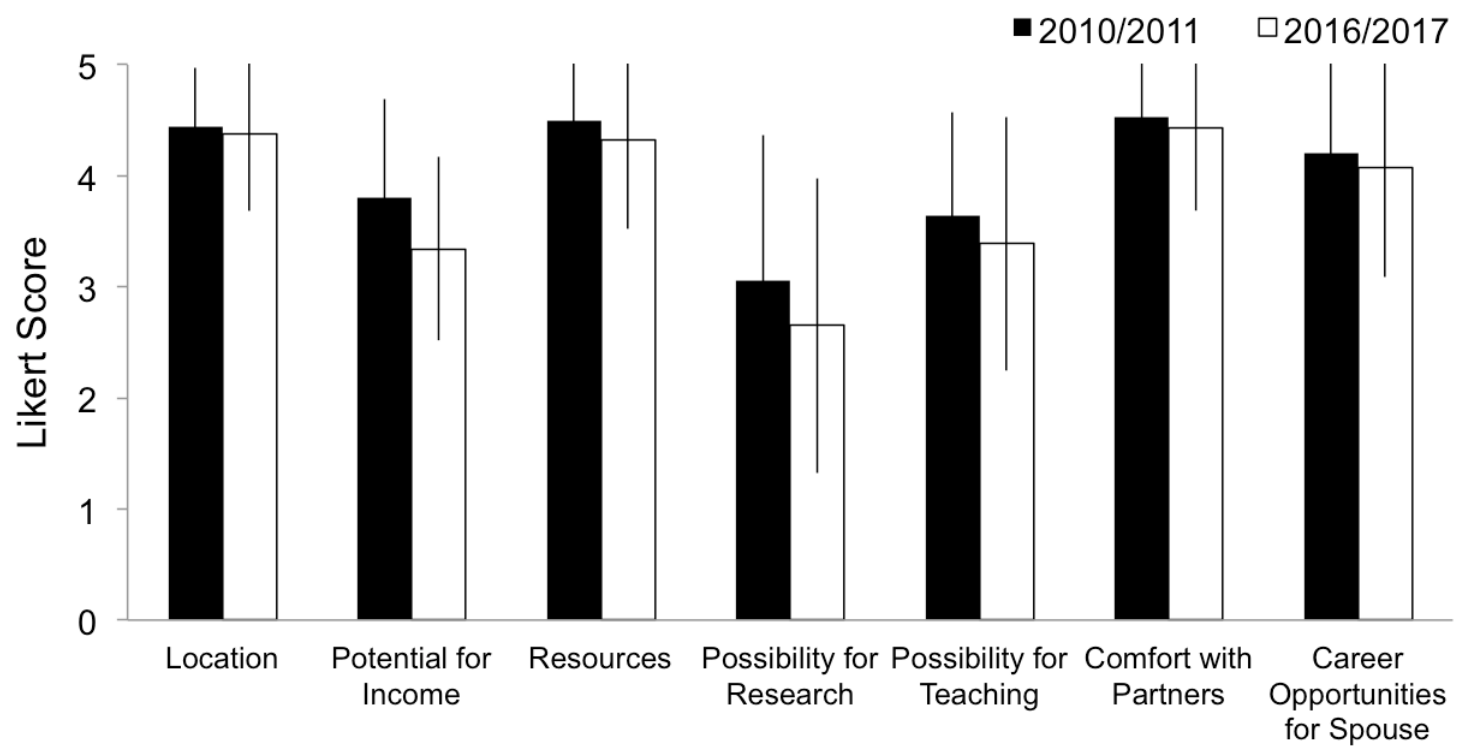


Fig. 5. Home province and desired location of practice for graduates in 2010/2011 and 2016/2017. There were no differences between the 2010/2011 and 2016/2017 cohorts. Provinces with net immigration included British Columbia, and Alberta. Provinces with net emigration were Saskatchewan/Manitoba, Quebec, and Newfoundland. Provinces with stability were Ontario, and Atlantic provinces.

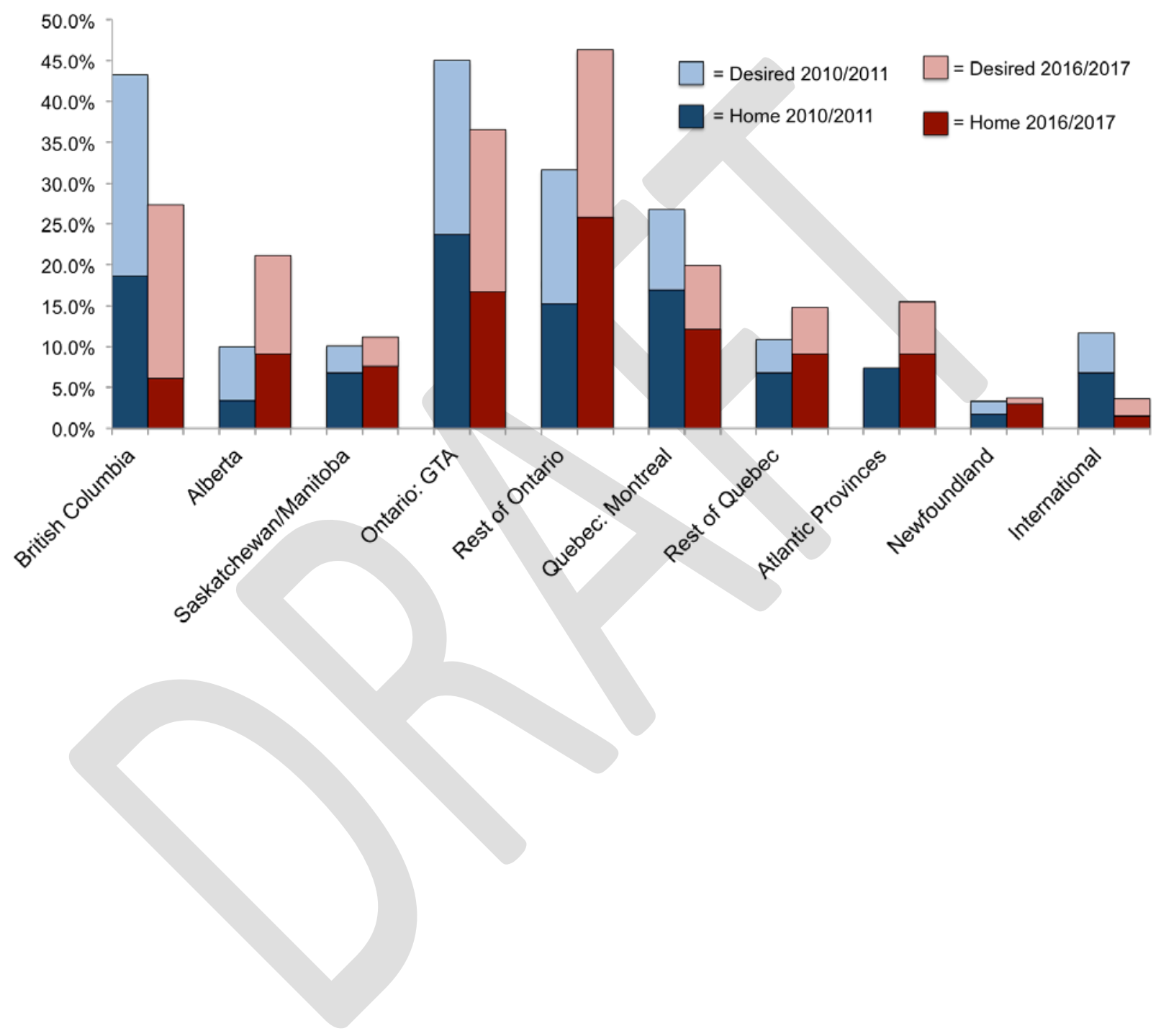

